\title{
Correction to: The initial assessment of expert panel performance in core hospitals for cancer genomic medicine in Japan
}

\author{
Kuniko Sunami ${ }^{1}$ - Yoichi Naito ${ }^{2}$ Eriko Aimono ${ }^{3} \cdot$ Toraji Amano $^{4} \cdot$ Daisuke Ennishi $^{5} \cdot$ Hidenori Kage $^{6} \cdot$ Masashi Kanai $^{7}$. \\ Keigo Komine $^{8} \cdot$ Takafumi Koyama $^{9} \cdot$ Takahiro Maeda $^{10}$. Sachi Morita ${ }^{11}$. Daisuke Sakai ${ }^{12}$. Shinji Kohsaka ${ }^{13}$. \\ Katsuya Tsuchihara ${ }^{14} \cdot$ Takayuki Yoshino $^{15}$
}

Published online: 30 March 2021

(c) Japan Society of Clinical Oncology 2021

\section{Correction to: \\ International Journal of Clinical Oncology \\ (2021) 26:443-449 \\ https://doi.org/10.1007/s10147-020-01844-1}

The author wishes to update the original publication as follows:

Under Results section of Abstract:

The sentence starting with: "A total of....." should read as:

A total of 747 cases underwent CGP tests, 28 cases (3.7\%) received genomically matched treatment, and 18 cases $(2.4 \%)$ were referred for genetic counseling.

In Results, under section: The performance of EPs at each core hospital:

The original article can be found online at https://doi.org/10.1007/ s10147-020-01844-1.

Takayuki Yoshino

tyoshino@east.ncc.go.jp

1 Department of Laboratory Medicine, National Cancer Center Hospital, Tokyo, Japan

2 Department of Breast and Medical Oncology, National Cancer Center Hospital East, Kashiwa, Japan

3 Genomics Unit, Keio University School of Medicine, Tokyo, Japan

4 Clinical Research and Medical Innovation Center, Hokkaido University Hospital, Sapporo, Japan

5 Department of Hematology and Oncology, Okayama University Hospital, Okayama, Japan

6 Department of Respiratory Medicine, The University of Tokyo Hospital, Tokyo, Japan

7 Department of Therpeutic Oncology, Graduate School of Medicine, Kyoto University, Kyoto, Japan

8 Department of Clinical Oncology, Tohoku University Hospital, Sendai, Japan
The sentence starting with: "Among the..." should read as:

Among the 747 cases in core hospitals, 28 cases $(3.7 \%$, range $0-7.7 \%$ ) received genomically matched treatments (Table 2);

The sentence starting with: "Regarding the..." should read as:

Regarding the germline findings, 18 of 747 cases $(2.4 \%$, range $0-15 \%$ ) were referred for genetic counselling (Supplementary Table 2).

In Table 4, the Recommended therapy for Site I should read as:

Alpelisib, neratinib

Publisher's Note Springer Nature remains neutral with regard to jurisdictional claims in published maps and institutional affiliations.

9 Department of Experimental Therapeutics, National Cancer Center Hospital, Tokyo, Japan

10 Department of Hematology, Oncology and Cardiovascular Medicine, Kyushu University Hospital, Fukuoka, Japan

11 Department of Clinical Oncology and Chemotherapy, Nagoya University Hospital, Nagoya, Japan

12 Center for Cancer Genomics and Personalized Medicine, Osaka University, Suita, Japan

13 Section of Knowledge Integration, Center for Cancer Genomics and Advanced Therapeutics, National Cancer Center, Tokyo, Japan

14 Division of Translational Informatics, National Cancer Center Exploratory Oncology Research and Clinical Trial Center, Tokyo, Japan

15 Department of Gastrointestinal Oncology, National Cancer Center Hospital East, 6-5-1, Kashiwanoha, Kashiwa-shi, Chiba 277-8577, Japan 